\title{
28 Resarch Square \\ Surgical management of acute limb ischemia, the first experience from Ethiopia
}

Nebyou Seyoum Abebe ( $\nabla$ nebyouss@yahoo.com )

Berhanu Daba Mekonnen

Addis Ababa University School of Medicine

Berhanu Nega Alemu

Addis Ababa University School of Medicine

Research article

Keywords: acute ischemia, thrombosis, embolism, revascularization, and limb amputation

Posted Date: January 7th, 2020

DOl: https://doi.org/10.21203/rs.2.20174/v1

License: (9) This work is licensed under a Creative Commons Attribution 4.0 International License.

Read Full License 


\section{Abstract}

Background Acute Limb Ischemia is a devastating emergency condition due to a sudden decrease in limb perfusion that threatens life or limb viability. It carries a high morbidity and mortality rate. This is the first experience to report on risk factors, etiologies and surgical outcomes of acute limb ischemia in Ethiopian.

Methods A Prospective Cross-sectional Study on patients operated for acute limb ischemia at Tikur Anbessa Specialized Hospital and Teklehaimanot General Hospitals from January 1, 2018, to December 31, 2018, Addis Ababa, Ethiopia was done. They were followed for 3 months to determine risk factors, etiologies and surgical outcomes of the disease. Data were collected using a structured questionnaire. Findings were analyzed using SPSS version 20 and compared with current literature.

Result A total of 102 patients were operated with a male to female ratio of 2:1 and the mean age at presentation was $54 \pm 17$ years. Hypertension, 40 (39.2\%), and diabetes mellitus, 32 (31.4\%), were the most common risk factors followed by combined hypertension \& diabetes mellitus, 20 (19.6\%). The most common cause of acute limb ischemia was thrombosis, 77 (75.5\%) and followed by embolism 14 (13.7\%) and trauma $11(10.8 \%)$. The source of emboli in all cases was cardiac disease due to atrial fibrillation, 9 (7.8\%), myocardial infarction, 6 (5.9\%) \& rheumatic valvular heart disease, 4 (3.9\%). The overall Presentation was very late with an average duration of $9 \pm 4.8$ days and all of them arrive after 24 hours of symptom onset. One hundred eight procedures were done in 102 patients. Type of procedure performed were, thrombectomy 51(47.2\%), primary amputation 24(22.2\%), bypass or interposition vascular grafts $10(9.2 \%)$, embolectomy $10(9.2 \%)$, primary vascular repair $7(6.4 \%)$, and Femoro-femoral graft $6(5.5 \%)$. The 30 -day amputation \& mortality rate was $52.9 \%$ and $9.8 \%$ respectively. The rate of limb loss after revascularization surgery was $32.4 \%$. Systemic complications occurred in $17.6 \%$ and local complications occurred in $35.3 \%$ of patients. On logistic regression analysis, age older than 60 years showed a four-fold risk of amputation $(p=0.017)$ and ten times the risk of mortality $(p=0.037)$ compared to 20-40 years age group. Hypertensive patients had 3 times more risk of amputation $(p=$ 0.031 ) as compared to non-hypertensive patients. The duration of presentation beyond 9 days showed 4 folds risk of amputation ( $p=0.021)$ as compared to those presented within 3 days. Previous Myocardial infarction was associated with 5 folds increase in mortality $(p=0.036)$ as compared to those without myocardial infarction.

Conclusion This review has tried to show the overall perspective of acute limb ischemia specific to the population we serve. Patients generally presented very late with a significant number of them arrived with irreversible ischemia and tissue loss. Age $\geq 60$ years; hypertension, previous myocardial infarction and delayed presentation were associated with poor surgical outcomes. An awareness to create the importance of early arrival, optimizing co-morbidities, timely detection and treating immediately on arrival of the patient, play a key role in improving surgical outcomes of acute limb ischemia. Keywords: Acute ischemia, Thrombosis, Embolism, Re-vascularization, Limb amputation

\section{Background}


According to the 2007 Transatlantic Intersociety Consensus (TASC II) for the management of PAD, Acute Limb Ischemia (ALI) is defined as a sudden decrease in limb perfusion that causes a potential threat to limb viability in patients who present within two weeks of acute onset of symptoms [1]. ALI is a medical emergency and it continues to have high morbidity and mortality. Despite urgent revascularization with thrombolytic, endovascular or open vascular surgery, the 30 days amputation and mortality rates range between $10-30 \%$ and $5-15 \%$ respectively $[2,3]$. This condition affects between $15-26$ persons per 100,000 each year and the associated morbidity and mortality is extremely high, with a yearly mortality rate of $40 \%$ in the USA $[1,3]$.

The commonest cause of ALI is PAD (85\%), where thromboses formed in atherosclerotic vessels or previously patent artery or graft. Embolus dislodged from distant sources accounts for $15 \%$ of nontraumatic cases $(2,3,4)$. Owing to lack of a valid survey in LMICs, a population-based estimate of patients with ALI is difficult. However, analysis of combined data from the Global Burden of Diseases program (5) and a systematic review of prevalence surveys (6) can describe the burden of PAD at global and regional levels (7). This population burden can be measured with the Disability Adjusted Life Year (DALY), which is a composite of the years of life lost owing to premature death caused by PAD and the years lived with disability owing to the disease. (5) Accordingly, the rates of DALYs were consistently greater in higherincome than in lower-income regions. However, the rate of growth between 1990 and 2010 was found greater in the lower-income regions, occurring in both men and women. These findings are consistent with those observed on the prevalence of PAD using the $A B I$, in which a marked increase was found between 2000 and 2010, more so in LMIC. (6) Similarly, analysis made on the Ethiopian national mortality burden during 1990-2015 showed that Ethiopia has successfully reduced premature mortality related to a communicable disease, however, diseases like cardiovascular, diabetes, cancer, and other non-communicable diseases have become the leading causes of death in 2015. (8)

This all shows that ALI has begun and will continue to be a disease burden to our community in the coming years. Besides, the disease impact on the occupation and quality of life of the individual patient is substantial, often resulting in loss of independence and/or lively hood. In Ethiopia, surgical services to treat ALI have been started recently within a few hospitals. Our experience in surgically treating ALI is limited; hence in order to significantly improve the surgical outcome and functionality of the limb, a proper understanding of the problem and its course of managements are essential. Besides, the unique demographic, Ethnic, and geographic factors that exist in our community could influence the disease progress and affect the surgical outcome which may require further investigations. To date, literature review showed that the majority of publications made so far were from developed countries, with very few reports from Africa and none from Ethiopia. Accordingly, we report our experience on the risk factors, etiologies, outcome and associated factors influencing the surgical outcome of ALI treated at Tikur Anbessa Specialized Hospital (TASH) and Teklehaimanot General Hospital.

\section{Materials And Methods}


Institutional based prospective cross-sectional study was done on all patients operated (limb amputation or revascularization surgery) for acute limb ischemia from January 1, 2018, to December 31, 2018, at TASH and Teklehaimanot General Hospitals, Addis Ababa, Ethiopia. TASH is the governmental tertiary center and Teklehaimanot General Hospital is a private center. Both of them are located in Addis Ababa, the capital city of Ethiopia and these hospitals were selected as the site of our study because most of the vascular surgery cases in Ethiopia are being managed in these institutions.

The study population included all patients who underwent surgery for acute limb ischemia at TASH and Teklehaimanot General Hospitals over a period of one year. Those patients with PAD without ischemia; chronic or critical limb ischemia, who were managed medically; patient disappeared before the intervention or refused surgery, and who had incomplete data set for analysis were excluded from the current study.

All patients were operated by Consultant Vascular Surgeon and were followed for a maximum of 3 months at the vascular clinic to determine risk factors, etiologies and surgical outcomes in terms of limb amputation/salvage, complications, and mortality. A total of 124 patients who presented with acute limb ischemia were seen during the study period and 116 of them underwent surgical procedures in an attempt to salvage limb and life. Of operated patients, 102 were eligible and included in the final analysis of this study. The rest of them were excluded as they do not meet inclusion criteria (3 refused surgery, 2 unfit for surgery, 3 medically treated, 14 of them had incomplete data set; of these, 8 lost from follow up \& 6 had incomplete investigations)

Study Variables

The measurable variables in this study were defined by reviewing similar literature previously published in acute limb ischemia. Accordingly, age, sex, risk factors of ALI and duration of presentation were used as independent variables and the dependent variables were surgical outcomes of ALI (limb amputation/salvage, local and systemic complications, mortality).

Data collection, Processing, and Analysis

Six personnel, principal investigator and 5 research assistants who were oriented on this study collected the data. They used a predesigned structured questionnaire that was prepared in English and translated into Amharic and Afan Oromo languages and translated back to English. The questionnaire was filled from the interview and medical records of patients prospectively. After data collection completed, cleaning, checking and editing of the content was done.

Then data was entered into the computer, data were analyzed using SPSS software version 20 and the results were expressed in a ratio, frequency and percentage were calculated using SPSS software version 20. And statistical significance test using logistic regression analysis (bivariate and multivariate) was applied to see the association between independent variables and dependent variables. $P$-value $<0.05$ was accepted significant and variables found to be significant on bivariate analysis were included in 
multivariate logistic regression analysis. Then the result was discussed by comparing analyzed data with other research done regionally and globally.

\section{Ethical Consideration}

This study was approved by and Research \& Ethical Committee of Addis Ababa University, College of Health Sciences. The permission was taken from archives of TASH and Teklehaimanot General Hospitals to retrieve a medical record of needed patients. Pre-consent oral counseling of participant and written informed consent was taken and confidentiality was protected at all steps.

\section{Results}

Of 102 patients operated for acute limb ischemia, 69 (67.6\%) of them were males and $33(32.4 \%)$ were females with male to female ratio being 2:1. The mean age at presentation was $54 \pm 17$ years (age ranges $23-95$ years) and 28 (27.5\%) cases occurred in $20-40$ years, 40 (39.2\%)cases in $40-60$ years \& 34 $(33.3 \%)$ cases in older than 60 years age groups.

The most common risk factors identified were Hypertension, 40 (39.2\%), diabetes mellitus, 32 (31.4\%), followed by combined hypertension \& diabetes mellitus, 20 (19.6\%) and smoking in 7 (6.9\%). (Table 1) Based on the diagnosis made using combination of clinical, imaging (Doppler U/S and/or CT Angiography) and intraoperative findings, the single most common etiology of ALI was found to be thrombosis, $77(75.5 \%)$ patients and in $46(45.1 \%)$ cases, thrombus occurred on the background of atherosclerosis. The other causes of ALI were embolism, 14 (13.7\%) and trauma in 11 (10.8\%) cases. The source of emboli was the heart in all patients in which 4 (3.9\%) patients had previous ischemic heart disease; 3 (2.9\%) patients had atrial fibrillation; 3 (2.9\%) patients had rheumatic valvular heart disease with atrial fibrillation. (Table 1 ) 
Table 1

Frequency of demographic factors, atherosclerotic \& cardiac risk factors that have Acute Limb Ischemia and the corresponding amputation rates

\begin{tabular}{|lllll|}
\hline Risk factors for ALI & No, (\%) & Amputation rate (\%) & P-value & OR \\
\hline Male sex & $69(67.6)$ & 38.2 & 0.596 & 1.2 \\
\hline Age $>$ 60 years & $34(33.3)$ & 19.6 & 0.017 & 3.75 \\
\hline Age 40-60 years & $40(39.2)$ & 26.5 & 0.032 & 3.04 \\
\hline Hypertension & $40(39.2)$ & 29.4 & 0.031 & 2.76 \\
\hline Diabetes Mellitus & $32(31.4)$ & 17.6 & 0.163 & 2.1 \\
\hline Hypertension \& Diabetes Mellitus & $20(19.6)$ & 13.7 & 0.028 & 10.1 \\
\hline Smoking & $7(6.9)$ & 3.9 & 0.478 & 1.8 \\
\hline Dyslipidemia & $4(3.9)$ & 1.9 & 0.999 & 1.1 \\
\hline Ischemic Heart Disease & $4(3.9)$ & 2.9 & 370 & 0.6 \\
\hline Atrial Fibrillation & $3(2.9)$ & 1.9 & 0.345 & 2.2 \\
\hline Ischemic Heart Disease \& & $2(1.9)$ & 1.9 & 0.761 & 1.6 \\
\hline Atrial fibrillation & $3(2.9)$ & 1.9 & 0.496 & 0.8 \\
\hline RVHD and atrial fibrillation & $1(0.9)$ & 0.9 & 0.703 & 0.5 \\
\hline Rheumatic Valvular Heart Disease & $1(0.9)$ & 0.9 & 0.703 & 0.5 \\
\hline Dilated Cardiomyopathy & & & & \\
\hline
\end{tabular}

The average duration of symptoms before presentation at the hospital was $9 \pm 4.8$ days (time ranges 115 days). All our patients presented after 24 hours of the onset of symptoms and 18 (17.6\%) patients presented within 1-3 days, 35 (34.4\%) patients within 4-9 days \& 49 (48\%) patients came to the hospital within 9-15 days. The predominant presenting symptoms of ALI were acute onset rest pain in the limb, 84 (82.5\%), limb swelling, 65 (63.7\%) and darkish discoloration of the limb in 47 (45.9\%) patients. On physical examination, the most common clinical signs were pulseless extremity in $73(71.6 \%)$, cold extremity in $68(66.7 \%)$ and swollen limb in $65(63.7 \%)$ patients. Of 102 patients came with ALI, 47 (45.9\%) of them had gangrenous limb and $58(56.9 \%)$ of them developed limb neurologic deficit (sensory and or motor) at presentation (Table 2). Of patients presented with gangrene, 43 (42.2\%) of them were treated with amputation ( 24 primary \& 19 secondary amputations) \& $4(3.9 \%)$ of them had digital gangrene, which sloughed off after re-vascularization. 
Table 2

Main symptoms and Physical findings of patients with Acute Limb Ischemia

\begin{tabular}{|lll|}
\hline Symptoms & No. & (\%) \\
\hline Rest extremity pain & 84 & 82.5 \\
\hline Limb swelling & 65 & 63.7 \\
\hline Limb darkish discoloration & 47 & 45.9 \\
\hline Limb reddish discoloration & 42 & 41.2 \\
\hline Numbness & 39 & 38.2 \\
\hline Physical Findings & & \\
\hline Pulseless & 73 & 71.6 \\
\hline Cold extremity & 68 & 66.7 \\
\hline Swollen limb & 65 & 63.7 \\
\hline Neurologic deficit & 58 & 56.9 \\
\hline Gangrene & 47 & 45.9 \\
\hline Limb hyperemia & 36 & 35.3 \\
\hline Compartment syndrome & 5 & 4.9 \\
\hline
\end{tabular}

Table 1. Frequency of demographic factors, atherosclerotic \& cardiac risk factors that have Acute Limb Ischemia and the corresponding amputation rates

In this study, the distribution of Acute Limb Ischemia showed anatomic predilection in which ischemic condition was more observed in lower extremities than upper extremities. Accordingly, 88 (86.3\%) patients had lower extremity involvement and out of these patients, 5 (4.9\%) of them had bilateral lower extremity while left lower extremity was predominant $45(44 \%)$. Of all patients, $14(13.7 \%)$ of them had upper extremity ischemia.

Our patients were followed for a maximum of 3 months and adherence to follow up was good ( $80 \%$ at 3 months). In our series, all patients were surgically managed with revascularization surgery or amputation. The most commonly performed procedure was thrombectomy in $51(47.2 \%)$ cases and out of these, 8 patients underwent thrombectomy with primary major amputation and 3 patients had thrombectomy with the reversed great saphenous vein bypass graft. Subsequently, on 30th day follow up; re-thrombectomy was done in $6(11.7 \%)$ patients. On the other hand, $24(22.2 \%)$ patients were treated with primary major amputation and embolectomy was performed in 10 (9.2\%) patients.

Table 2. Main symptoms and Physical findings of patients with Acute Limb Ischemia 
The primary vascular repair was performed in 7 (6.4\%) for partial or complete traumatic vascular transection. In the rest of our patients, $5(4.6 \%)$ reversed great saphenous vein (hereafter GSV) interposition graft for complete traumatic vascular injury and $5(4.6 \%)$ reversed GSV bypass graft for acute thrombosis occurred in atherosclerotic vessels. Six (5.5\%) extra-anatomic crossover femorofemoral bypass graft was performed for unilateral common \& external iliac thrombosis. The overall amputation rate following each procedure is described in Table 3 below.

Table 3

Performed surgical procedures for ALI and the corresponding subsequent amputation rates in 3 months follow up

\begin{tabular}{|lll|}
\hline Primary Surgical Procedure & Number (\%) & Amputation rate (\%) \\
\hline Thrombectomy & $51(47.2)$ & 17.6 \\
\hline Primary major amputation & $23(21.3)$ & \\
\hline Embolectomy & $10(9.2)$ & 3.9 \\
\hline Primary vascular repair & $7(6.4)$ & 0.9 \\
\hline $\begin{array}{l}\text { Extra-anatomic crossover } \\
\text { Femoro-femoral graft (PTFE) }\end{array}$ & $6(5.5)$ & 3.9 \\
\hline Interposition graft (Reversed GSV) & $5(4.6)$ & 1.9 \\
\hline Bypass graft (Reversed GSV) & $5(4.6)$ & 3.9 \\
\hline Primary minor amputation & $1(0.9)$ & \\
\hline Total & $108(100)$ & \\
\hline
\end{tabular}

In this study, the 30 -day amputation rate was $52.9 \%$ (8.8\% minor \& $44.1 \%$ major amputation) while the limb salvage rate was found to be $37.1 \%$ (Table 5). In general, the amputation rate after an attempt at revascularization was $32.4 \%$. During this period, 36 (35.3\%) patients developed procedure-related complications in which re-thrombosis of native vessels and surgical site infections were the commonest complications accounting for $14.7 \% \& 12.6 \%$ respectively. On the other hand, systemic complications occurred in $18(17.6 \%)$ patients and the most common systemic complications were acute coronary syndrome in $6(5.9 \%)$ and acute kidney injury in $5(4.9 \%)$ cases (Table 4). Of all patients, $10(9.8 \%)$ patients died during first 30 days follow up and the predominant causes of death being: an acute coronary syndrome in $6(5.9 \%)$, overwhelming sepsis of surgical wound focus in $3(2.9 \%)$ \& acute respiratory insufficiency in $1(0.9 \%)$ patients. In this study, the 30 -day amputation rate was $52.9 \%(8.8 \%$ minor \& $44.1 \%$ major amputation) while the limb salvage rate was found to be $37.1 \%$ (Table 3 ). 
Table 4

Procedural and Systemic complications of Acute Limb Ischemia patients who underwent surgical procedures in 3 months follow up

\begin{tabular}{|llll|}
\hline Morbidity & Complications & $\begin{array}{l}\text { At 30th day } \\
\text { No. (\%) }\end{array}$ & $\begin{array}{l}\text { At 3rd month } \\
\text { No. (\%) }\end{array}$ \\
\hline Systemic complications & Acute coronary syndrome & $6(5.9)$ & $6(5.9)$ \\
\cline { 2 - 4 } & Acute kidney injury & $5(4.9)$ & $5(4.9)$ \\
\cline { 2 - 4 } & Sepsis & $3(2.9)$ & $3(2.9)$ \\
\cline { 2 - 4 } & Cerebrovascular attack & $3(2.9)$ & $4(3.9)$ \\
\cline { 2 - 4 } & Acute respiratory insufficiency & $1(0.9)$ & $1(0.9)$ \\
\hline Procedure related complications & Re-thrombosis of native vessels & $15(14.7)$ & $20(19.6)$ \\
\cline { 2 - 4 } & Surgical site infection & $13(12.6)$ & $15(14.7)$ \\
\cline { 2 - 4 } & Graft failure & $6(5.9)$ & $6(5.9)$ \\
\cline { 2 - 4 } & Others & $2(1.9)$ & $2(1.9)$ \\
\hline Total & & $48(47.1)$ & $40(39.2)$ \\
\hline
\end{tabular}

Table 5

Comparison of amputation rates and functional status of the salvaged limb at 30th day \& 3rd month follow up of patients operated for ALI

\begin{tabular}{|llllll|}
\hline Limb outcome parameters & & \multicolumn{3}{l}{ At 30th day } & \multicolumn{2}{l|}{ At 3rd month } \\
\hline Amputation rate & NO. & $\%$ & No. & $\%$ \\
\hline Primary amputation & Major & 23 & 22.5 & 23 & 22.5 \\
\cline { 2 - 6 } & Minor & 1 & 0.9 & 1 & 0.9 \\
\hline Secondary amputation & Major & 21 & 20.6 & 22 & 22.5 \\
\cline { 2 - 6 } & Minor & 9 & 8.8 & 11 & 10.8 \\
\hline Limb salvage rate with or without minor amputation & & & & \\
\hline Pulse status & Improved & 41 & 40.2 & 40 & 39.2 \\
\cline { 2 - 6 } & Unchanged & 5 & 4.9 & 4 & 3.9 \\
\hline Functional status & Normal & 39 & 38.2 & 39 & 38.2 \\
\cline { 2 - 6 } & Claudication & 5 & 4.9 & 4 & 3.9 \\
\hline
\end{tabular}


Table 3. Performed surgical procedures for ALI and the corresponding subsequent amputation rates in 3 months follow up

In general, the amputation rate after an attempt at revascularization was $32.4 \%$. During this period, 36 (35.3\%) patients developed procedure-related complications in which re-thrombosis of native vessels and surgical site infections were the commonest complications accounting for $14.7 \%$ \& $12.6 \%$ respectively. On the other hand, systemic complications occurred in 18 (17.6\%) patients and the most common systemic complications were acute coronary syndrome in $6(5.9 \%)$ and acute kidney injury in $5(4.9 \%)$ cases (Table 4). Of all patients, 10 (9.8\%) patients died during first 30 days follow up and the predominant causes of death being: an acute coronary syndrome in 6(5.9\%), overwhelming sepsis of surgical wound focus in $3(2.9 \%)$ \& acute respiratory insufficiency in $1(0.9 \%)$ patients.

Table 4. Procedural and Systemic complications of Acute Limb Ischemia patients who underwent surgical procedures in 3 months follow up

In 3 months follow up, one other patient died of overwhelming sepsis of wound focus and 3 more patients got amputation following Fogerty catheter thrombectomy after a 30-day postoperative period (see Table 5). Amputation and mortality rates became 55.9\% \& 10.8\% respectively on 3 months follow up. In this study, few patients developed additional systemic and procedural complications beyond 30 postoperative days (see Table 4). So, a total of 44 (43.2\%) procedural and 19 (18.6\%) systemic complications occurred in 3 months postoperative period.

On logistic regression bivariate analysis, age more than 60 years was found to be significantly associated with increased rate of $\operatorname{limb}$ amputation $(p=0.017 ; \mathrm{OR}=3.8 ; 95 \% \mathrm{Cl}, 1.26$ to 11.11 ) and mortality $(\mathrm{p}=$ $0.037 ; \mathrm{OR}=9.72 ; 95 \% \mathrm{Cl}, 1.15$ to 82.32 ) compared to $20-40$ years age group. Significantly more patients with combined hypertension and diabetes mellitus underwent amputation $(p=0.028 ; \mathrm{OR}=10.15 ; 95 \% \mathrm{Cl}$, 1.29 to 80.12 ) compared to patients who had neither hypertension nor diabetes mellitus. Similarly, patients with hypertension alone showed significant association with increased risk of amputation $(\mathrm{p}=$ $0.031 ; \mathrm{OR}=2.76 ; 95 \% \mathrm{Cl}, 1.05$ to 6.32 ) compared to non- hypertensive.

Table 5. Comparison of amputation rates and functional status of the salvaged limb at 30th day \& 3rd month follow up of patients operated for ALI

In the same way, the duration of symptoms before presentation at hospital had strong association with increased risk of amputation and patient who came after 9 days (average time in this study) underwent more amputation ( $p=0.013 ; \mathrm{OR}=4.22 ; 95 \% \mathrm{Cl}, 1.36$ to13.12) than those who presented within 3 days but no significant association was found between duration of presentation and mortality rate. A significant rate of mortality ( $p=0.036 ; O R=5.31 ; 95 \% \mathrm{Cl}, 1.11$ to 25.38$)$ was observed in patients who had Ischemic heart disease compared to those who didn't have it.

However, patients with diabetes mellitus alone, smoking, dyslipidemia and cardiac risk factors (Ischemic heart disease, atrial fibrillation, rheumatic valvular heart disease, dilated cardiomyopathy) were 
statistically tested separately \& in combination but didn't show any significant difference in limb amputation rate (see Table 1) or mortality rate. There was no statistically significant independent variable found to be associated with the rate of occurrence procedural or systemic complications. To reduce the effect of confounding factors, logistic regression multivariate analysis was done and the only finding was patients who presented after 9 days had a significant rate of amputation $(p=0.021 ; 0 R=4.73 ; 95 \%$ $\mathrm{Cl}, 1.27$ to 17.69$)$ compared to first 3 days

\section{Discussions}

Despite improvement in treatment modalities like urgent revascularization with thrombolytic or vascular surgeries (the advent of endovascular or open vascular procedures), ALI continues to be a common problem of LMICs that carries high morbidity and mortality. $(1,3)$ With the current study, we have found out that ALI is coming to be one of the major NCDs and patients generally presented very late with a significant number of them arrived with irreversible ischemia and tissue loss. Age $\geq 60$ years; hypertension, previous myocardial infarction and delayed presentation were found to be significantly associated with poor surgical outcomes.

In this study, we found a predominance of ALI in males (67.6\%), which was almost twice as common as in females (32.4\%). Even though this finding is slightly higher, it is a consistent with other studies [20, 24, 27]. The mean age at presentation was $54 \pm 17$ years, which was similar to data of other reports. [27, 32] Patients within age 40-60 years were commonly affected (39.2\%) and followed by those older than 60 years (33.3\%). Most authors agree that ischemic conditions of lower extremities are more common than those of upper extremities $[11,15,16,20]$. In research done in India (Tertiary center) in 2008, it was also shown that acute lower extremity ischemia was more common than acute upper extremity ischemia [27]. In these studies, the forwarded explanation for these findings is an unequal distribution of atherosclerotic occlusive diseases, mostly affecting lower limbs. In our study, we found favorably similar findings to this literature where lower extremity involvement accounted for $86.3 \%$ and upper extremity in $13.7 \%$ cases. There was bilateral involvement in $4.9 \%$ cases and left lower extremity was predominantly involved (44\%).

Among risk factors identified in our study, atherosclerosis, hypertension and diabetes mellitus were the most common accounting for $39.2 \%$ and $31.4 \%$ respectively while smoking $(6.9 \%)$ and dyslipidemia (3.9\%) were the least contributing risk factor. Similarly, Johannes M. et al (2014) and Mazda T. et al (2008) ascertained that hypertension and diabetes mellitus were common risk factors for ALI and in contrary to our findings, they found cigarette smoking as the most predisposing factor for acute limb ischemia compared to these two comorbidities $[24,27,30]$. In support of this evidence, many studies have shown an association of hypertension, diabetes mellitus and smoking with the development of PAD (atherosclerosis). The relative risk of developing PAD is two to three folds for smoking \& hypertension. So currently hypertension guidelines support aggressive treatment of blood pressure in a patient with atherosclerotic occlusive diseases, targeting a blood pressure of $140 / 90 \mathrm{mmHg}$ or $130 / 80 \mathrm{mmHg}$ should be targeted in patients with diabetes or renal insufficiencies $[22,23,25,26]$. 
In the current study, the main etiologies of acute limb ischemia identified were thrombosis $(75.5 \%)$ followed by embolism (13.7\%) and trauma (10.8\%). The source of embolism in all cases was from the heart. Previous myocardial infarction (3.9\%), atrial fibrillation (2.9\%), rheumatic valvular heart diseases with atrial fibrillation (2.9\%) and ischemic heart disease with atrial fibrillation (1.9\%) were common cardiac causes of embolism identified. Similar findings were reported from India, West Africa and other areas $[2,3,4,27,32]$. Unlike our report, a research article done in University of Michigan (USA), Kaunas University of Medicine Hospital (Lithuania) and Aims Shams University (Egypt) reviewed their single institutional experiences and found embolism as more frequent cause of ALI than acute thrombosis. Such discrepancy was seen because of higher number of patients with cardiac diseases in their community and the availability of improved diagnostic and therapeutic modalities for cardiac patients in their community that allowed for better survival than ours where such facilities are scarcely available [24, $31,33]$.

As opposed to trends seen in other series, in our study, all patients with ALI presented after 24 hours duration, which was beyond the accepted critical cutoff time for limb ischemia (golden hour of 4-6 hours) [8]. Unlike most of the reports done in other countries, the average duration of symptoms before arrival to the hospital was $9 \pm 4.8$ days. Because of this late presentations, our patients presented rest extremity pain (82.5\%), limb swelling (63.7\%) and darkish discoloration of limb (45.9\%). On physical examination, pulseless extremity (71.6\%), cold extremity (66.7\%) and swollen limb (63.7\%) were the most common clinical signs. In our analysis of 102 patients, $45.9 \%$ of them had gangrenous limb and more than one-half of patients developed neurologic deficit (sensory, motor or both) at presentation, the figure, which was higher than the report from Iran by M. Mozzafar A. et al [28]. This implicates most of our patients had a significant delay in presentation and had a late stage of limb ischemia which was responsible for high morbidity and mortality in our set up.

On subsequent 3 months follow up, these patients showed different outcomes in terms of limb salvage, local and systemic postoperative complications. Accordingly, amputation rates (secondary amputations) following thrombectomy, vascular grafts (of all types) and embolectomy were $17.6 \%, 7.8 \%$ \& $3.9 \%$ respectively while $8.8 \%$ of patients underwent re-thrombectomy for an indication of native vessel rethrombosis in 3 months progress. The 30 -day amputation rate was $52.9 \%$ with a limb salvage rate of $37.1 \%$. Three more patients underwent amputation on subsequent 3 months follow up and overall amputation rate became $55.9 \%$ with a limb salvage rate of $33.3 \%$. This figure was two to three folds higher than the results in other studies [27-32]. The rate of limb loss following revascularization procedure was $32.4 \%$, this figure was higher than data from other studies $[29,30,32]$.

In our analysis, we observed that primary vascular repair and reversed GSV interposition graft done for the partial or complete vascular injury had better limb salvage rate (8.8\%) compared to bypass graft (limb salvage rate of $2.9 \%$ ) performed for other causes of ALI but the association was not statistically significant. The reason for this was attributed to the relatively early presentation of trauma patients and prompt intervention that might have salvaged the limb in this group of patients. However, reversed GSV and PTFE bypass grafts done for ALI showed almost comparable results in terms of limb salvage rate. 
The amputation rate after bypass grafts in our set up was much lower than a trend in another study as described by Donald T. Baril et al [29] even though the comparison was difficult as few patients underwent bypass procedure in our series.

The 30-day mortality rate of ALI in our study was $9.8 \%$ and only one other patient died in 3 months follow up making an overall mortality rate of $10.8 \%$. We found acute coronary syndrome (5.9\%), overwhelming sepsis of surgical wound focus (2.9\%) and acute respiratory insufficiency $(0.9 \%)$ as common causes of death in our set up. The mortality rate in our series was much lower (two folds) than a report from other authors [31, 32] but comparable with review made in Iran and Lithuania [28, 33]. We also observed that acute coronary syndrome (5.9\%), acute kidney injury (4.9\%) and sepsis of surgical wound focus were common systemic complications while re-thrombosis of native vessels (14.7\%), surgical site infections (12.6\%) and graft failure (5.6\%) were procedural related postoperative complications. In general, systemic complications occurred in $17.6 \%$ and procedural related complications occurred in $35.3 \%$ of patients in 30 postoperative days, the higher figure compared to experience from South Africa [30].

Another important observation of this study was that the effect of independent factors on morbidity and mortality in ALI was evaluated. Accordingly, age older than 60 years was associated with almost three folds increase in limb loss and about nine folds increase in mortality compared to those within 2040 years of age. Similarly, hypertensive patients showed about three times higher risk of amputation than those without hypertension in our analysis. There were ten folds increase in limb loss in patients having combined hypertension and diabetes compared to those who had neither of them. Jonathan L.E et al described in their single institutional experience that diabetic patients had the four-folds risk of amputation compared to non-diabetic patients [24] and it was also observed that smokers have twice the risk of limb loss compared to non-smokers in another study [27]. In contrast to this, there was no significant difference found in diabetic or smoker patients in terms of amputation or mortality rate in our analysis.

Among risk factors of $\mathrm{ALI}$, in addition to older age, ischemic heart disease showed a strong association with mortality rate \& had five times the risk of mortality compared to those without cardiac ischemia. Surprisingly, there was no statistically significant independent factor associated with postoperative complications in this series. In our final model of analysis (multivariate), it was observed that the duration of symptoms before presentation above nine days was associated with five folds increase in limb loss rate compared to the first three days.

From this evidence, we can conclude that a much higher rate of limb loss in this study was attributed to delayed presentation of patients with ALI and associated late stage of limb ischemia \& this finding was asserted by many other authors $[27,28,32,34]$. So, we strongly suggest that the early arrival of patients, timely detection and early intervention play a vital role in the success of the management of ALI. We did not investigate the pattern of referral in our study and difficult to give a concrete explanation for the delayed presentation of patients. However, our speculation as reasons for delayed presentation could be financial problems, the remoteness of vascular centers and lack of health awareness of patients, 
attention, and level of awareness of referring physicians about this disease entity. We hope this will be the main area of study for the subsequent researcher.

This study has some limitations. First, Some patient's data were found to be incomplete and few patients also lost from follows up so they were excluded from our analysis. The gap in this database could result in selection bias. Second, the duration of follow up is only 3 months hence the long-term effect is not known. Third, as this study was conducted at only two institutions, it may be difficult to generalize the results to the national level. Hence, the national-based study may address this issue in the subsequent research.

\section{Conclusion}

The causes of acute limb ischemia were thrombosis, embolism or trauma in our institutions and had significantly high morbidity and mortality. Among risk factors of ALI, age older than 60 years, hypertension, combined hypertension \& diabetes mellitus, myocardial infarction and delayed presentation were associated with poor surgical outcomes (high rate of amputation and mortality). Therefore, treating and optimizing comorbidities, the early arrival of the patient, timely detection and intervention play a key role in improving surgical outcomes of acute limb ischemia.

In an attempt to decrease this high rate of morbidity and mortality resulting from ALI, we should strengthen the awareness of referring health professionals at all levels on this particular issue. Finally, we recommend that the role of anticoagulants in changing the outcome of ALI and the pattern of referral of this disease should be the area of focus on subsequent research.

\section{Abbreviations}

- ALI

Acute limb ischemia

- PAD

Peripheral arterial disease

- TASH

Tikur Anbessa specialized hospital

- LMICs

Low middle income countries

- PTFE

Polytetrafluoroethylene

- GSV

Great saphenous vein

Funding

\section{Declarations}




\section{Acknowledgment}

We would like to acknowledge the assistance of Addis Ababa University, College of Health Sciences, and the Department of Surgery in undertaking this research. We also like to thank the members of the record room staff at Tikur Anbessa Specialized Teaching and Teklehaimanot General Hospitals for their undue cooperation. We would like to thank Addis Ababa University postgraduate librarians and medical science librarians for providing journals, allowing Internet access and providing books while required We are grateful to Dr. Adugna Tasew (MD, MPH) and Dr. Zerihun Kassa (MD, MPH) who supported us by providing additional comments, helping us during analyzing data, preparing, editing and writing this research paper.

Funding: Not applicable

\section{Author's contribution}

1) Nebyou S. Abebe- Study concept and design, data interpretation, article writing, final approval, accountability for all aspects of the work.

2) Berhanu D. Mekonnen - Data collection, analysis and interpretation, final approval, accountability for all aspects of the work

3) Berhanu N. Alemu - Data analysis, interpretation, Critical revision, drafting, and final approval, accountability for all aspects of the work

All authors read and approved the final manuscript.

Ethics approval and consent to participate: This study was approved by and Research \& Ethical Committee of Addis Ababa University, College of Health Sciences. The permission was taken from archives of TASH and Teklehaimanot General Hospitals to retrieve a medical record of needed patients. Pre-consent oral counseling of participant and written informed consent was taken and confidentiality was protected at all steps. A copy of the written consent is available for review by the Editor-in-Chief of this journal

Consent for Publication: Not applicable

Availability of data and material: A soft copy of all data used for this article is available with the corresponding author

Competing Interests: The authors declare that all authors in its form approved this manuscript and that no competing interest exists.

Confirmation: We confirm that the content of the manuscript has not been published, or submitted for publication elsewhere. 


\section{References}

1. Varu VN, Hogg ME, Kibbe MR, Critical limb ischemia; J Vasc Surg, 2010 Jan: 51(1): 230-41. doi: 10.1016/j.jvs.2009.08.073 2. Davies MG, O'Malley K, Feeley M, Colgan MP,Moore DJ, Shanik G; Upper limb embolus: a timely diagnosis. Ann Vasc Surg; 1991 Jan: 5(1): 85-7 , DOI:10.1007/BF02021785 ; PMID:1997084 3. Blaisedel, FW, Steele M, Allen RE; management of acute lower limb ischemia due to embolism and thrombosis; Surgery; 1978 Dec; 84(6): 822-34; PMID:715701 4. Savelyev VS, Zatevakhin MD, Steanov MD. Artery embolism of the upper limbs; Surgery; 1977Apr : 81(4):367-75 PMID: 8476435. Murray, C. J. et al. Disability-adjusted life years (DALYs) for 291 diseases and injuries in 21 regions, 19902010: a systematic analysis for the Global Burden of Disease Study 2010. Lancet; 2012: 380: 21972223. 6. Fowkes, F. G. et al. Comparison of global estimates of prevalence and risk factors for peripheral artery disease in 2000 and 2010: a systematic review and analysis. Lancet; 2013: 382:1329-1340 7. Sampson, U. K. et al. Global and regional burden of death and disability from peripheral artery disease: 21 world regions, 1990 to 2010. Glob. ; Heart; 2014; 9:145-158.e21. 8. Awoke M, Gizachew A, Yohannes A, et al, National mortality burden due to communicable, non-communicable, and other diseases in Ethiopia, 1990-2015: findings from the Global Burden of Disease Study 2015; Popul Health Metr. 2017; 15: 29. PMCID: PMC5521057; PMID: 28736507 ; doi: 10.1186/s12963-017-0145-1 9. Creager MA, Kaufman JA, Conte MS; Clinical Practice. Acute limb ischemia; New Engl J Med. 2012 Jun; 7; 366(23): 2198-206. doi:10.1056/NE JMCP 1006054 10. Largiader, J. and E. Schneider, Therapy of acute peripheral arterial occlusion; Herz, 1991. 16(6): 456-62. 11. Henke, P.K., Contemporary management of acute limb ischemia: factors associated with amputation and in-hospital mortality. Semin Vasc Surg, 2009.22(1): 34-40. 12. SantistranJr, O'Connell, J.B., W.J. Quinones-Baldrich, Proper evaluation and management of acute embolic versus thrombotic limb ischemia. Semin Vasc Surg; 2009:22(1): 10-6. 13.Eliason JC. WainessRM, proctor mc, Dimick JB, cown JA Jr ,upchurch GrJr,etal,A national and single institutional experience in the contemporary treatment of ALEl. Annsurg; 2003; 2038:382-9 14. Ueberrueck T, Marusch F, Schmidt H, Gastinger I. Risk factors and management of arterial emboli of the upper and lower extremities; J Cardiovasc Surg (Torino). 2007 Apr; 48(2): 181-6; PMID: 17410064 15. Hughes K, Hamdan A, Schermerhorn M, Giordano A, Scovell S, Pomposelli F Jr. Bypass for chronic ischemia of the upper extremity: results in 20 patients. J Vasc Surg. 2007 Aug; 46(2): 303-7; DOl:10.1016/j.jvs.2007.04.035 16. Eyers P, Earnshaw JJ ; Acute non-traumatic arm ischemia. Br J Surg. 1998 Oct; 85(10): 1340-6; DOI:10.1046/j.1365-2168.1998.00884.x 17. Quraishy MS, Cawthorn SJ, Giddings AE.; Critical ischemia of the upper limb; J R Soc Med 1992; 85(5):269-73 ; PMCID:PMC1294603 18. Norgeren L, Hiatt WR, Dormandy JA; Inter-society consensus for the management of peripheral arterial disease. Eur $\mathrm{J}$ VascEndovascSurg 2007; 33:S1-74 19. Pentti J, Salenius JP, Kuukasjärvi P, Tarkka M. Outcome of surgical treatment of acute upper limb ischaemia. Ann Chir Gynaecol. 1995 ; 84(1): 25-8; PMID:7645905 20. Haimovici H. Cardiogenic embolism of the upper extremity. J CardiovascSurg 1982 MayJun:23(3):209-13; PMID:7085739 21. Management of Peripheral Arterial Disease (PAD) Trans-Atlantic Intersociety Consensus (TASC). J VascSurg 2000; 31(1 part 2): S1-287. 22. Selvin E, Marinopoulos S, Berkenblit G, Rami T, Brancati FL, Powe NR, et al. Meta-analysis: glycosylated hemoglobin and cardiovascular disease in diabetes mellitus. Ann Intern Med 2004; 141(6): 421-31. 23. Eliason JL, Waines 
RM, Proctor MC et al. Contemporary treatment in acute lower limb ischemia. Ann Surg; 2003 Sep; 238(3): 382-90. PMCID: PMC1422711 DOI:10.1097/01.sla.0000086663.49670.d1 24. Chobanian AV, Bakris GL, Black HR, , et al. Seventh report of the Joint National Committee on Prevention, Detection, Evaluation and Treatment of High Blood Pressure; Hypertension 2003 Dec; 42(6): 1206-52. PMID: 14656957 DOI:10.1161/01.HYP.0000107251.49515.c2 25. Bonaa K, Njolstad I, Ueland P, Schirmer H, Tverdal A, Steigen $\mathrm{T}$. et al. Homocysteine lowering and cardiovascular events after acute myocardial infarction. $\mathrm{N}$ Engl J Med 2006; 354:1578-88. 26. Mazda T, Prabhu Pk, Edwins, Sunil A. management of acute limb ischemia. The national medical journal of india, 2008; 21(6) p. 284-7 27. M. Mozaffar, A. Afsharfard, F. Malekpour, R. Vaghardoost. Embolectomy for Acute Lower Limb Ischemia. Medical Journal of the Islamic Republic of Iran, 2004; 18(2): p.131-4. 28. Donald T. Baril, Virendra I. Patel, Dejah R et al. Outcomes of lower extremity bypass performed for acute limb Ischemia. J Vasc Surg.; 2013 October; 58(4): 949-956. 29. Johannes MT, NG Niadoo. Upper limb ischemia. Vascular surgery, 2014; p. 61-82 30.Sherif S, Denis, Ahmed Setal. atraumatic acute limb ischemia. Vasculary surgery, 2001.35:p.3 31.Yangni-Angate $H$, Adoubi A, Adoh Adoh M, Yapobi Y, Coulibaly AO, et al. ; Acute non-traumatic limb ischemia; West Afr J Med , 2006 Apr-Jun; 25(2):101-4; PMID:16918179 32. Aleksandras A. and Nerijus A. the surgical treatment of acute lower limb ischemia. MEDICINA (2003) ; 39 (7): p.646-653. 33. Fagundes C, Fuchs FD, Fagundes A, Poerschke RA, Vacaro MZ. Prognostic factors for amputation or death in patients submitted to vascular surgery for acute limb ischemia. Vasc Health Risk Manag.;2005; 1(4): 345-9.

PMCID:PMC1993960 DOI:10.2147/vhrm.2005.1.4.345 